\title{
1 Accessibility Analysis of Risk Severity
}

2 Mengying Cui

3 University of Minnesota

4 Department of Civil, Environmental, and Geo- Engineering

5500 Pillsbury Drive SE

6 Minneapolis, MN 55455 USA

7 cuixx242@umn.edu

8 David Levinson

9 RP Braun-CTS Chair of Transportation Engineering

10 Director of Network, Economics, and Urban Systems Research Group

11 University of Minnesota

12 Department of Civil, Environmental, and Geo- Engineering

13500 Pillsbury Drive SE

14 Minneapolis, MN 55455 USA

15 dlevinson@umn.edu

$164040+6$ figures +2 tables $=6040$ words

17 July 31, 2015 


\section{ABSTRACT}

2 Risk severity in transportation network analysis is defined as the effects of a link or network fail-

3 ure on the whole system. Change accessibility (reduction in the number of jobs which can be

4 reached) is used as an integrated indicator to reflect the severity of a link outage. The changes of

5 accessibility before-and-after the removing of a freeway segment from the network represent its

6 risk severity. The analysis in the Minneapolis - St. Paul (Twin Cities) region show that links near

7 downtown Minneapolis have relative higher risk severity than those in rural area. The geographical

8 distribution of links with the highest vulnerability displays the property that these links tend to be

9 near or at the intersection of freeways. Risk severity of these links based on the accessibility to

10 jobs and to workers at different time thresholds and during different dayparts are also analyzed

11 in the paper. The research finds that network structure measures: betweenness, straightness and

12 closeness, help explain the severity of loss due to network outage. 
1 INTRODUCTION

2 The ability of freeway networks to deal with the variances or failures of certain nodes or links is

3 important for efficient operation. Risk severity analysis allows us to measure that ability.

Risk severity expresses the consequences of a failure node, link or network on the operation efficiency of transportation network as a whole. Combined with the likelihood of failure (which we do not address here), we can understand the vulnerability of a link, and how much resources should be devoted to ensuring link outages are avoided. Vulnerability can be seen as the inverse of network reliability, which is defined as the probability that people or goods can successfully move from one place to another. That means a road network with higher reliability has a relative lower vulnerability for a transportation system.

In the recent years, variety of attributes are used to measure reliability focusing on different aspects of transportation. Connectivity reliability were firstly proposed due to the damage of road network resulting from the nature disasters(1)(2). It illustrates whether the road network is connected or not regardless of other traffic attributes, such as travel time(3). Travel time and capacity are also proposed as the reliability measures considering the degradation of the road network(4). Travel time reliability stands for the probability that a trip between a certain OD pair could reach the destination successfully within a specific time interval(5). It describes the temporal uncertainty that travelers experienced during their trips in a road network, which is a fundamental factor for both transportation efficiency and travel behavior(6). While capacity reliability were proposed as the probability that the capacity of a road network could satisfy with the travel demand at a required level of service considering the travel behavior, specifically the route choice behavior(1)(7).

The two objectives of vulnerability analysis are to identify the critical network components, such as links or nodes, which could cause the worst consequences for the transportation system if they failed (8), and the likelihood of such failure. There are many different definitions of road network vulnerability.

Berdica used serviceability to measure the consequences of incidents, and proposed that vulnerability could be treated as a susceptibility to incidents, which shows the extent of serviceability reduction caused by the incidents (9). Both probability and consequences of the failures determine the vulnerability based on the definitions. Hence, to reduce vulnerability, a fail-safe approach, which means to reduce the probability of the fails, and a safe-fail approach, which means to reduce the negative effects result from the fails, are proposed (9). Nicholson and Du proposed a similar definition, which also considered the probability of risks (10).

D'Este and Taylor defined vulnerability by measuring the accessibility changes, concerning themselves only with the consequences of road network degradation but not probability of failure (4)(11) . A node would be vulnerable if the degradation of a small number of links could not significantly affect the accessibility of the node, while a link would be critical if the degradation of this link could significant affect the accessibility of the whole network (12). They used the Hansen integral accessibility index and developed a measure they called the Accessibility/Remoteness Index of Australia (ARIA) to measure the vulnerability of Australia National Transport Network (12). Taylor also used these two index to measure the regional network vulnerability of the Green Triangle Region in Australia (13).

In this study, we use accessibility to jobs and accessibility to workers as the indices to measure the risk severity of freeways in the Minneapolis - St. Paul region. The paper is organized as follows. Section 3 and section 4 show the methodology and data that we used in vulnerability measurement. The measurement results and analysis are presented in section 5. We summarize 
1 and conclude in section 6.

\section{DATA}

3 There are three different data sources are applied into the vulnerability analysis: TomTom speed

4 data and the linked road network, 2010 Transportation Analysis Zone (TAZ) System and the LEHD 5 origin-Destination Employment Statistics (LODES).

TomTom speed data and the linked TomTom road network in Twin Cities is the first dataset, which provide the basic geographical and transport information for the research. And the data were acquired from TomTom by the Metropolitan Council. TomTom speed data is a dataset, which contains speed data that were aggregated and processed based on GPS data with high spatial coverage on the road network, which shows the great spatial structure of their speed measurement. The GPS data reflect driving patterns of drivers and are used to develop speed profiles for each road segment (14).

According to the Functional Roadway Classifications, TomTom speed data were categories into four sections, from FRC0 to FRC4 (15). For each classification, the speed data were also separated based on different time periods of a day considering the traffic properties, such as peak hours (Morning and evening), non-peak hours(daytime and overnight). Moreover, for each group of time period, speed value was measured on different percentiles from 5 percent to 95 percent, in which 5th percentile speed stands for the highest speed category that only 5 percent of the drivers drive faster than, and the 95th percentile speed is the lowest speed category.

Since the vulnerability of freeways is the objective in this research, major arterials in Twin Cities were chosen to analyze the vulnerability based on the FRCs, and the total number of links is around 4,000. Moreover, accessibility changes in the before-and-after scenarios are the indexes to evaluate the vulnerability. Hence, we chose the median speed (50th percentile speed) of morning peak hours (7AM-9AM) to process the network analysis for searching the shortest travel time path.

The second data source is the 2010 Transportation Analysis Zone (TAZ) system in Twin Cities developed by the Metropolitan Council. This data is a polygon shapefile that contains geographic information of 7 counties in the Twin Cities region (16).

The third data source is the LODES data. It belongs to the Longitudinal Employer-household Dynamics(LEHD) data, which was obtained from the United States Census Bureau (17). The primarily used data is the Residence Area Characteristics (RAC). RAC provides the information about the total number of jobs considering the people who are living in the residential block. This data are the major resources for calculating the accessibility to workers.

\section{METHODOLOGY}

Based on Taylor's definition of vulnerability, a link would be critical if its degradation could significant affect the accessibility of the whole network (18). Then for a link in the freeway, its risk severity could be measured as the accessibility changes before and after the failure of the link:

$$
D_{l}=A-A_{l}
$$

where $D_{l}$ stands for the accessibility changes. $A$ is the weighted accessibility based on the complete road network, while $A_{l}$ is the weighed accessibility when link $l$ was removed from the network.

Weighed accessibility is an integrated index, which combines the accessibility values of all 
1 the zones by using the population as the weights. Hence, the expression of the weighted accessi-

2 bility is shown as,

$$
A=\frac{\sum_{i=1}^{n} A_{i} P_{i}}{\sum_{i=1}^{n} P_{i}}
$$

where $A_{i}$ stands for the accessibility of the ith zone, while $P_{i}$ is the population of that zone.

We focused on commute patterns, and thus measured accessibility to jobs in the morning peak hours, and the accessibility to workers in the evening peak hours in this study.

To measure the accessibility to jobs and to workers, we used the cumulative opportunity measure (19) (20). The basic idea for this measure is to count the number of opportunities (jobs, workers) that can be reached within a given time threshold (19). The accessibility based on the cumulative opportunity measure is calculated as:

$$
\begin{gathered}
A_{i}=\sum_{j} O_{j} f\left(C_{i j}\right) \\
f\left(C_{i j}\right)= \begin{cases}1 & \text { if } C_{i j} \leq T \\
0 & \text { if } C_{i j}>T\end{cases}
\end{gathered}
$$

Where $O_{i}$ stands for the opportunity ( jobs or workers in zone $j$ ), while $C_{i j}$ is the travel time on the shortest travel time path between origin $i$ and destination $j$. $T$ is the predetermined time threshold.

A time-weighted accessibility index is developed to combine the accessibility by time-ofday. The time-weighted accessibility is be expressed as,

$$
A=\frac{\sum_{i=1}^{7} A_{i} T i}{\sum_{i=1}^{7} T_{i}}
$$

where $A_{i}$ is the accessibility to jobs in the $i^{t h}$ time period of a day, while $T_{i}$ is the average number of trips per hour in the corresponding time period.

To compute the $A_{l}$, ArcGIS was used to obtain the shortest travel time path with and without the availability of link $l$ in the network, and calculate the travel time for each OD pair at the TAZ level for each scenario. In the current network definitions of Transportation Analysis Zones (TAZ2010), there are 2485 zones in the 7 counties in the study area. SQL Server was used to calculate the job accessibility by joining the employment and population information of each zone with the predetermined time threshold. Based on the TomTom network, there are around 4,000 freeway link segments in the network for the study area.

Note this analysis assumes travel speeds remain unchanged when links are removed, so does not account for subsequent congestion effects, if any.

\section{RESULTS}

The risk severity measurement for the freeway segments in the Twin Cities are shown in Figure 1, in which links with higher risk severity are visualized as red while those with lower risk severity are shown in blue. The time thresholds for the accessibility calculation in Figure 1 are 20 minutes.

Figure 1 shows that the freeway segments with relative higher risk severity tend to be in or near the downtown area. And these links are more likely to be located on I-94 and I-35W. More- 


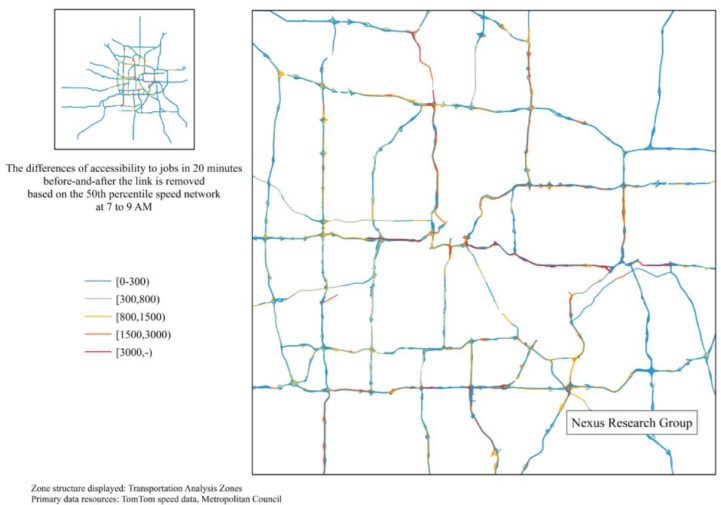

(a) Risk severity measurement results based on accessibility to jobs during morning peak hours

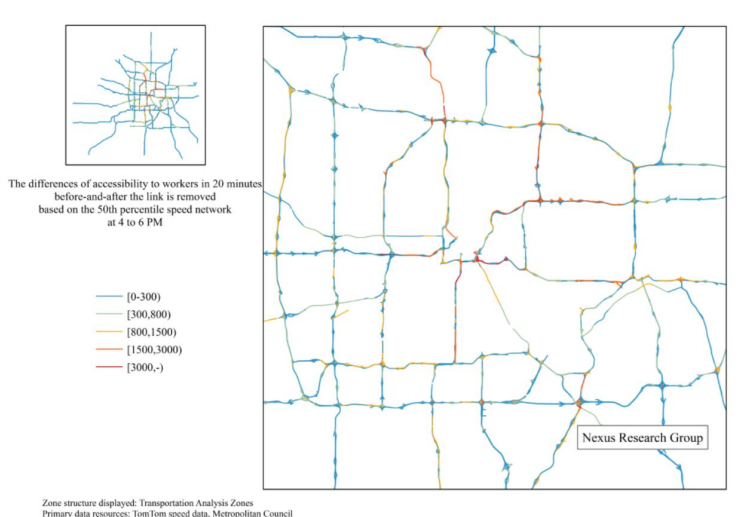

(b) Risk severity measurement results based on accessibility to workers during evening peak hours

FIGURE 1 : Risk Severity Measurement

1 over, there is no evident different pattern using accessibility to jobs and accessibility to workers as

2 the index to measure risk severity.

\section{Links with highest risk severity}

Using accessibility to jobs and accessibility to workers, the links with the highest risk severity differ. For accessibility to jobs, the link is located at the intersection of I-94 and Mn 280, which is displayed as the blue line in Figure 2 (b). While, for the latter one, the most vulnerable link is located at the intersection of I-35W and I-94, showing as the blue line in Figure 2 (d). And the accessibility changes for the former one (8474) is larger than the latter one (5343).

Figure 2 describes the comparison results of accessibility based on complete network and network without the links with the highest risk severity. From Figure 2 accessibility measurements for both access to jobs and to workers have the same distribution pattern. The zones with higher accessibility are centered on the downtown area, which is shown in red, and the accessibility decreases with the increasing of distance from downtown Minneapolis, which is visualized as the gradual change of color from red to green.

The differences before-and-after the links with the highest risk severity are removed from the network are evident for job accessibility (Figures $2 b$ and $2 d$ ). For accessibility to workers, the obvious changes based on the maps are only shown in the red area, which is near the removed link. But the lost accessibility (5343) is still notable.

Such significant differences are not surprising for two reasons. First, both links are located near downtown Minneapolis, which serves more traffic than suburban and rural area. Second, both of the two links are near the intersection of two freeways (I-94 and Mn 280, and I-35W and I-94), which connect more paths.

To further illustrate the last discussion, we also pick 20 links, which have the highest vulnerability, to clarify if they have the similar properties. The locations of these links are shown as red lines in Figure 3.

Obviously, considering both accessibility to jobs and accessibility to workers, all the links are located in or near the downtown area (downtown Minneapolis and downtown St.Paul) and most 


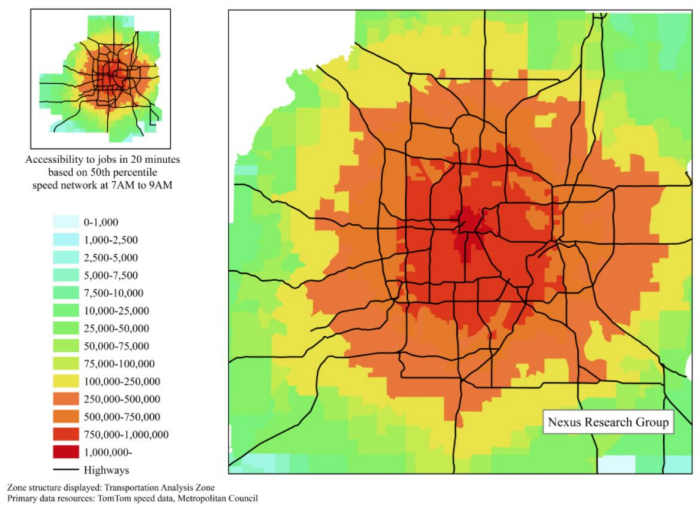

(a) Job accessibility based on complete network

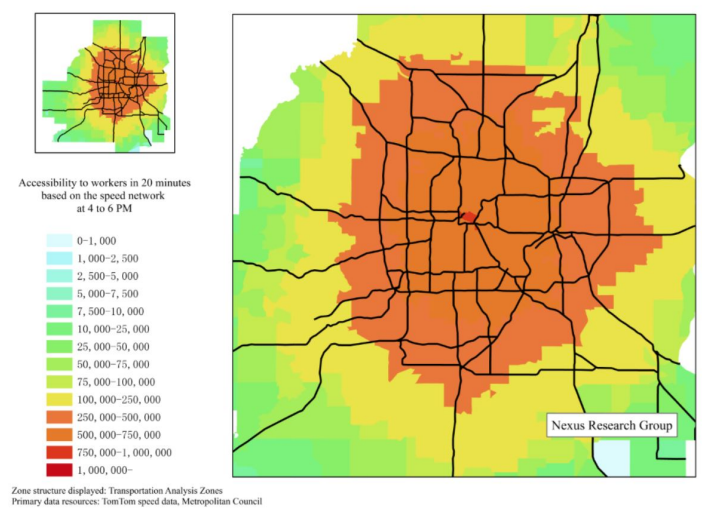

(c) Accessibility to workers based on complete net- $($ d) work

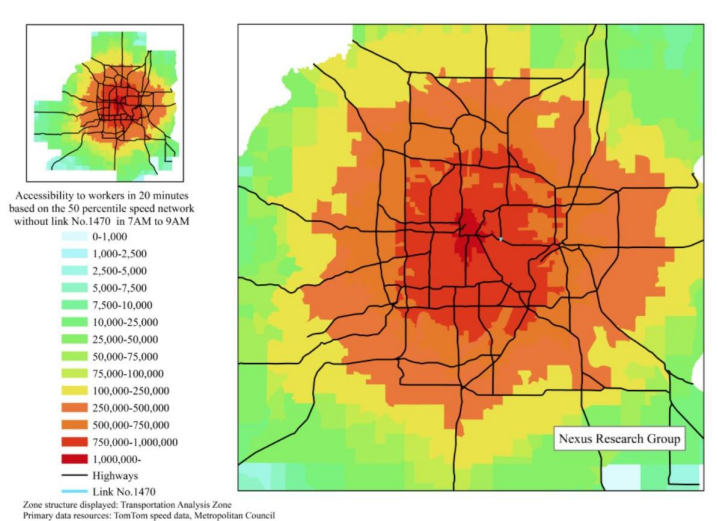

(b) Job accessibility based on the network with the link with the highest risk severity removed
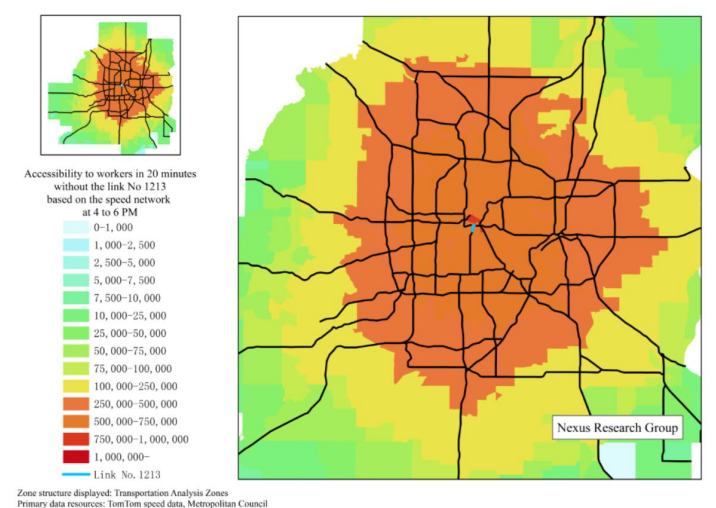

(d) Accessibility to workers based on the network with the link with the highest risk severity removed

FIGURE 2 : The difference of accessibility between complete network and incomplete network

1 of them are near or at the intersection of two different freeways, which further shows the existence

2 of the properties for the links with highest risk severity. However, the spatial distribution of these

3 top 20 links with the highest vulnerability in Figures ?? and ?? differ. For the morning, most of

4 the links in the Top 20 are distributed on I-94 and I-494, while for the afternoon, the Top 20 links

5 are distributed more widely on the freeway network.

\section{Vulnerability analysis by time thresholds}

7 The predetermined time threshold is a significant attribute in accessibility measurement. Hence, to

8 clarify how the predetermined time threshold affects the vulnerability analysis, we also calculated

9 the vulnerability based on the accessibility are 30 and 40 minutes thresholds. The comparison

10 considering both accessibility to jobs and accessibility to workers are shown in Figure 4.

Comparing the risk severity patterns in different time thresholds, it is shown that the links

12 with higher risk severity also have the similar pattern that most of them are near or in the downtown area for all the time thresholds we used. However, the freeway network seems to be more sensitive for vulnerability when the time thresholds are set as 20 minutes and 30 minutes since the the maps 


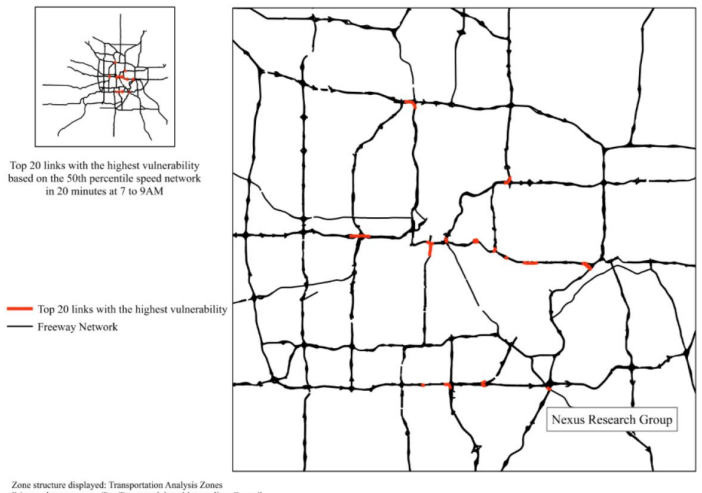

(a) Locations of 20 links with the highest vulnerability considering AM accessibility to jobs

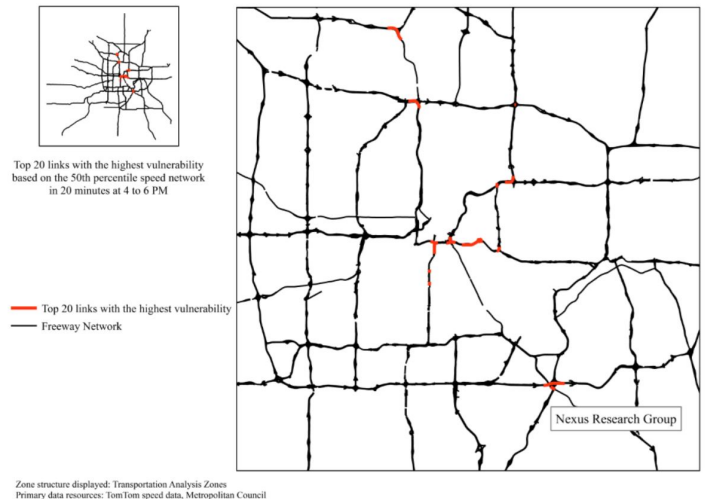

(b) Locations of 20 links with the highest vulnerability considering PM accessibility to workers

FIGURE 3 : Locations of 20 links with the highest vulnerability

TABLE 1 : Commuting trips by auto and accessibility by time of a day

\begin{tabular}{|l|l|l|l|l|l|l|l|}
\hline & $\begin{array}{l}\text { 10PM- } \\
\text { 5AM }\end{array}$ & $\begin{array}{l}\text { 5AM- } \\
7 \text { AM }\end{array}$ & $\begin{array}{l}7 \text { AM- } \\
\text { 9AM }\end{array}$ & $\begin{array}{l}\text { 9AM- } \\
\text { 2PM }\end{array}$ & $\begin{array}{l}\text { 2PM- } \\
\text { 4PM }\end{array}$ & $\begin{array}{l}\text { 4PM- } \\
\text { 6PM }\end{array}$ & $\begin{array}{l}\text { 6PM- } \\
\text { 10PM }\end{array}$ \\
\hline $\begin{array}{l}\text { Average No. } \\
\text { of trips }\end{array}$ & 4,369 & 182,487 & 352,542 & 87,679 & 44,912 & 26,888 & 7362 \\
\hline $\begin{array}{l}\text { Accessibility } \\
\text { to Jobs }\end{array}$ & 577,546 & 602,464 & 557,627 & 580,279 & 566,267 & 527,995 & 575,580 \\
\hline
\end{tabular}

1 in Figures $4 \mathrm{e}$ and $4 \mathrm{f}$ are more blue and the links are harder to be distinguished. Travelers can likely

2 reach most part of the cities in 40 minutes even in the absence of many selected links, and choose 3 other routes successfully without using the removed freeway links.

Similarly, the top 20 links with the highest risk severity at different time thresholds are also picked up for both accessibility to jobs and accessibility to workers, which are shown in Figure

6 5. As discussed before, in 20 minutes, these top 20 links are located near or in the intersection of

7 two different freeways around the downtown area. From Figure 5, the top 20 links show similar 8 patterns at 30 and 40 minutes thresholds.

However, differences remain. For accessibility to jobs, the locations of the top 20 links have the trends to move toward the southern part, which is farther from the downtown Minneapolis, from I-94 to I-494. These Top 20 links are clustered. For accessibility to workers, these top 20 links move farther from downtown Minneapolis area, but are less clustered. 


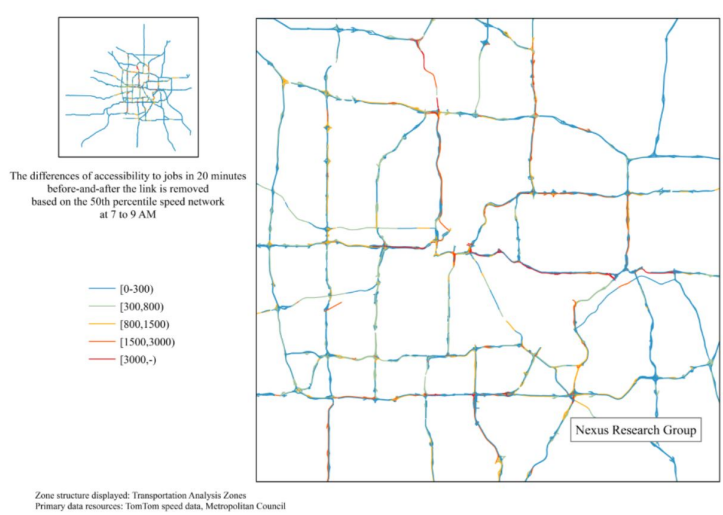

(a) 20 minutes

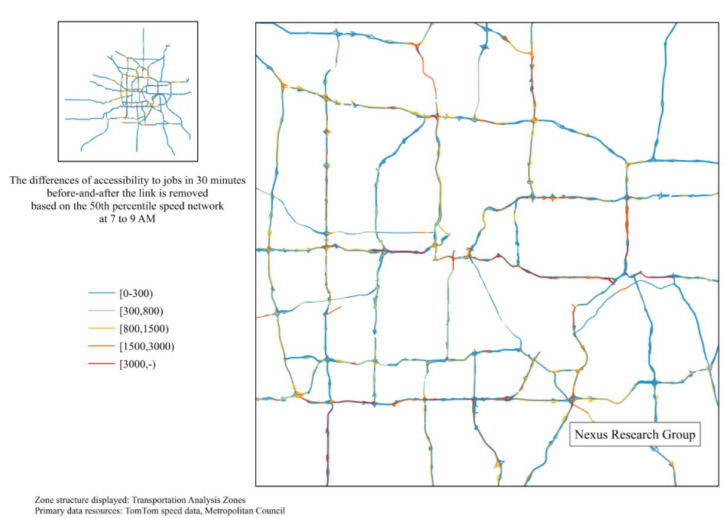

(c) 30 minutes

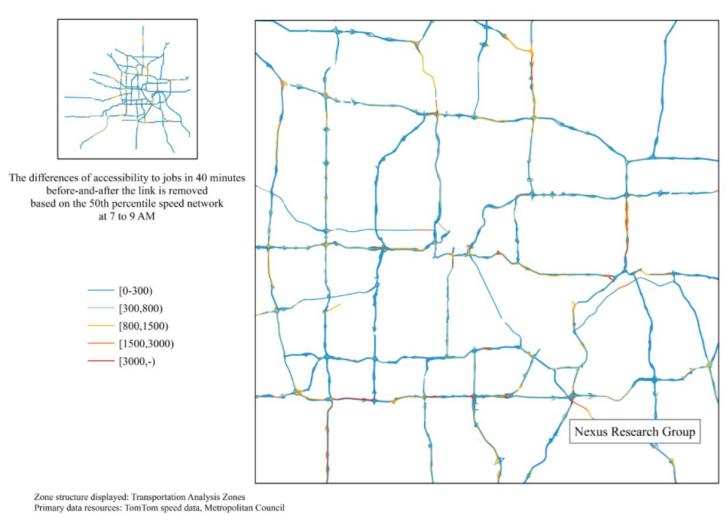

(e) 40 minutes

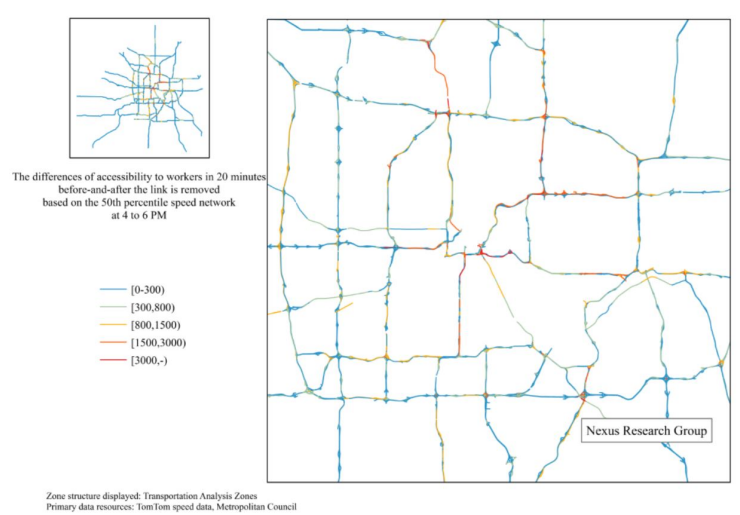

(b) 20 minutes

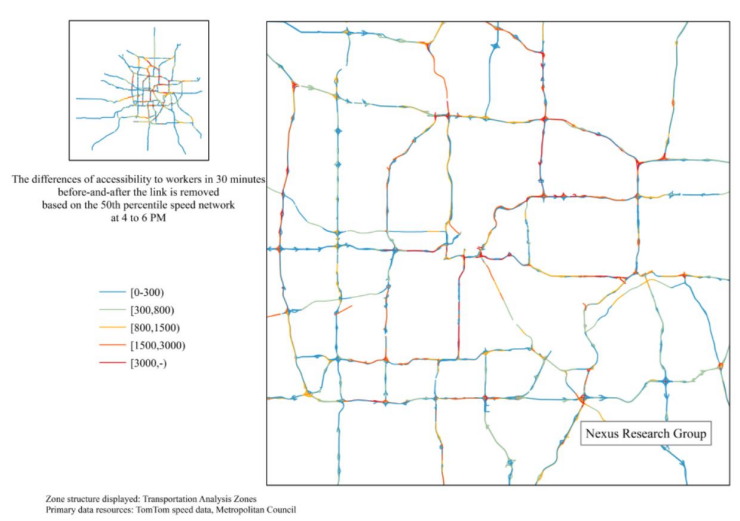

(d) 30 minutes

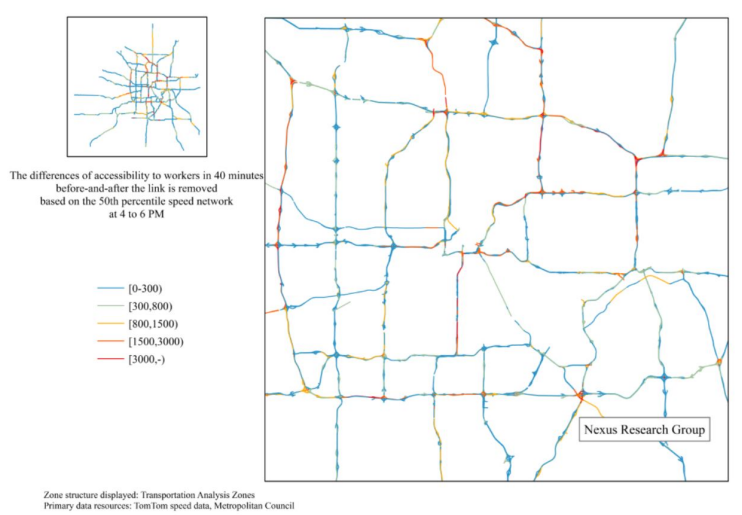

(f) 40 minutes

FIGURE 4 : Risk severity analysis results in different time threshold (The figures in the first line are based on the accessibility to jobs, while the figures in the second line are based on the accessibility to workers) 


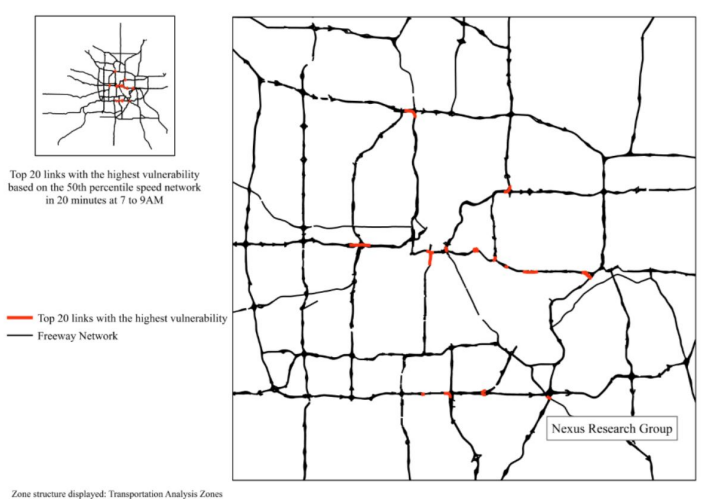

(a) 20 minutes

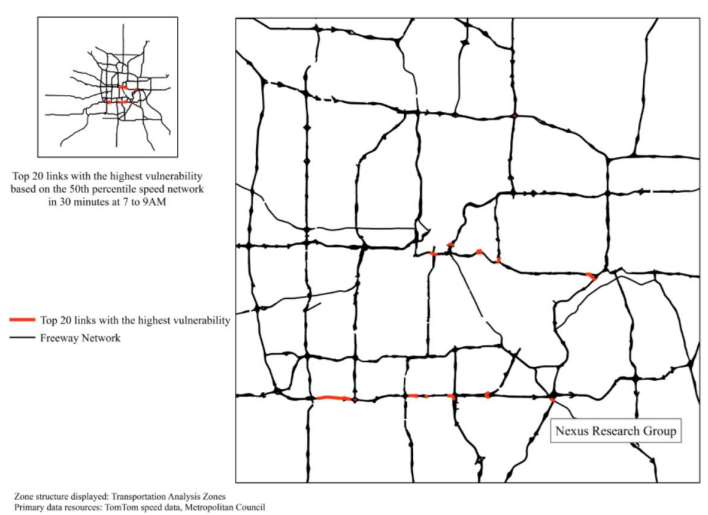

(c) 30 minutes

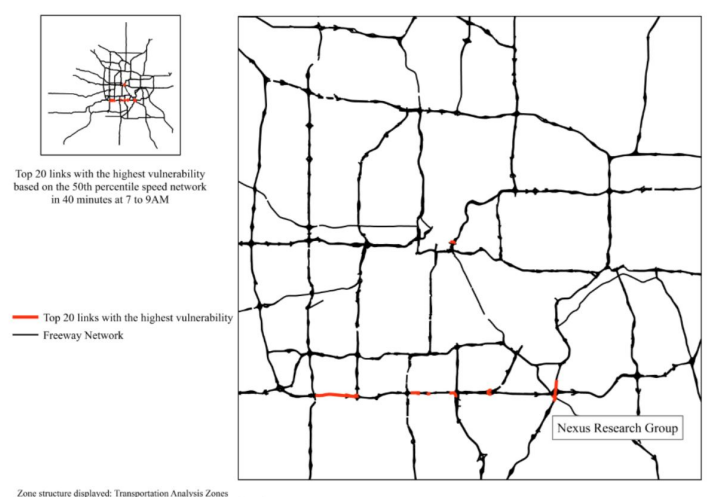

(e) 40 minutes

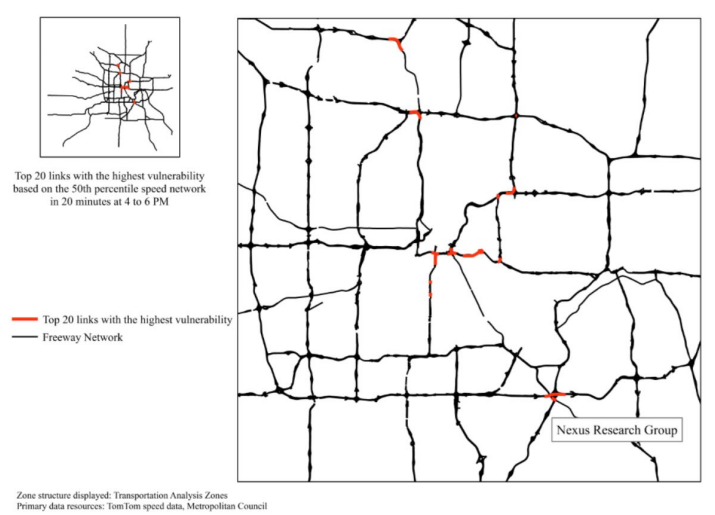

(b) 20 minutes

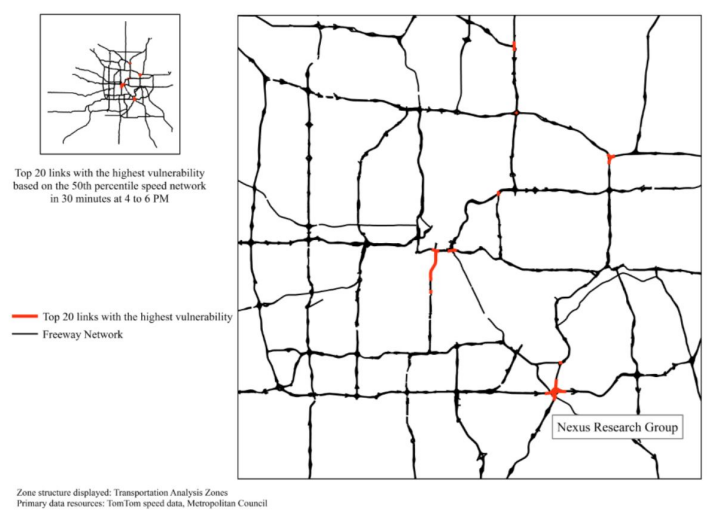

(d) 30 minutes

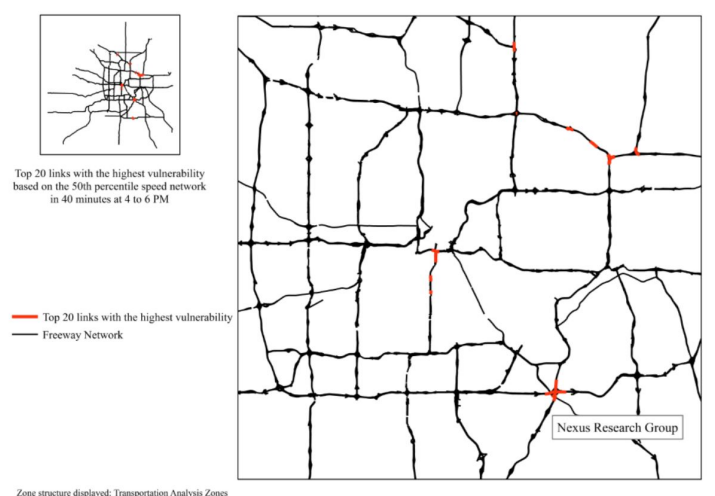

(f) 40 minutes

FIGURE 5 : Top 20 links with the highest risk severity in different time threshold (The figures in the first line are based on the AM accessibility to jobs, while the figures in the second line are based on the PM accessibility to workers) 


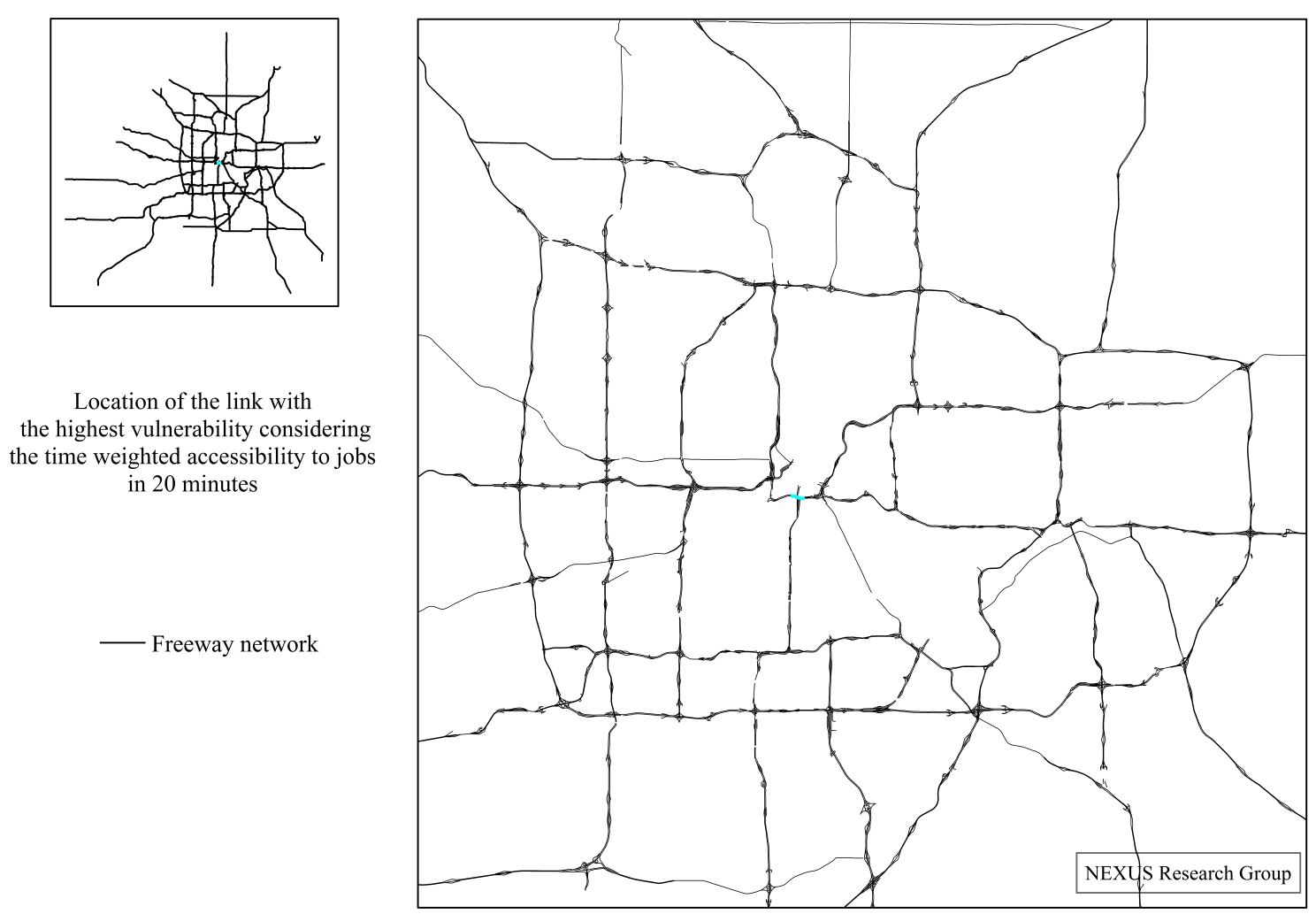

Primary Data Sources: TomTom Speed Data, Metropolitan Council

FIGURE 6 : The location of the link with the largest changes of time-weighted accessibility to jobs 
The data on number of trips was collected from the TBI2010. Trips by auto to work were chosen and used to calculate the average number of commute trips per hour. Based on the average number of trips per hour, the time-weighted accessibility based on the complete network is 571,756 .

Considering the computational burden of computing this analysis for multiple time parts for the large number of freeway segments, we only picked the top 20 links with the highest vulnerability in 20 minutes to calculate their time-weighted accessibility. The changes of the time-weighted accessibility stand for the corresponding risk severity. According to the results, the link with the highest risk severity based on the time-weighted accessibility is located in the intersection of I-94 and I-35W, shown as the blue dot in Figure 6.

\section{Regression of vulnerability on network structure measures}

Network structure refers to the topology of the network, which explains the network arrangement and connectivity of its elements, like nodes and links(21). Effective network structure measures could assist in understanding the details of network elements and their contribution to the whole network (22).

Previous researches confirmed that network structure could not only affect the performance of transportation system (congestion, daily vehicle kilometers traveled(DVKT) per capita, journeyto-work time, automobile mode share, and so on) (23)(24), but influence individual travel behavior (25). Risk severity, as an attribute showing the importance of network elements for the transportation system, is hypothesized to have a relationship with network structure. Many variables could be used to characterizing network structures. Betweenness, straightness and closeness are selected in this paper to examine their specific relationship with risk severity, which is discussed as following(26).

Betweenness quantifies the times of a node or a link in the network that is passed by the shortest paths between OD pairs (27). In this paper, the node or link represents the freeway segment. Hence, the betweenness of a freeway segment could be expressed as

$$
\text { Betweenness }_{i}=\sum_{j, k} \frac{n_{j k}(i)}{n_{j k}}
$$

where Betweenness $s_{i}$ stands for the betweenness measure for freeway segment $i . n_{j k}$ is the number of shortest paths from $j$ to $k$, while $n_{j k}(i)$ is the number of shortest paths from $j$ to $k$ that pass through freeway segment $i$.

Straightness describes the differences between the shortest network distances and the Euclidean distances, which is also called as the efficiency centrality (28)(29). It could be defined as

$$
\text { Straightness }_{i}=\sum_{j, k} \frac{\delta_{j k}}{d_{j k}}
$$

where Straightness $s_{i}$ represents the straightness measure for freeway segment $i . \delta_{j k}$ is the Euclidean distance between origin $j$ and destination $k$, while $d_{j k}$ is the shortest network distance between the OD pair. A similarly measure of the spatial efficiency is the network circuity, which is similar to the straightness based on the definition(30). The difference is that the network straightness is defined as the fraction of the sum of the shortest network distances and that of the Euclidean distance(23), 
1 which is expressed as

$$
\text { Circuity }_{i}=\frac{\sum_{j, k} d_{j k}}{\sum_{j, k} \delta_{j k}}
$$

$$
\text { Closeness }_{i}=\frac{1}{\sum_{i, j} d_{i j}}
$$

5 where $d_{i j}$ is the network distance of the shortest path between $i$ and $j(31)$.

In particular, it is hypothesized that the betweenness and straightness should have positive correlations, while closeness should have a negative one, based on the definition of these three measures(26). Hence, in this part, we analyze the effects of betweenness, straightness and closeness on risk severity based on accessibility to jobs at morning peak hours and accessibility to workers at evening peak hours by time threshold and test the hypotheses.

The Urban Network Analysis toolbox for ArcGIS, which is developed by City Form Lab, was used to collect the network structure measures for freeway links(26). And for different time thresholds, we chose the searching radius as 20,30 and 40 minutes respectively. The results of linear regression of vulnerability on betweenness, straightness and closeness are shown in Table 2.

\begin{tabular}{|c|c|c|c|c|c|c|c|}
\hline & \multicolumn{3}{|c|}{ Morning Peak Hours } & \multicolumn{3}{|c|}{ Afternoon Peak Hours } \\
\hline & & $20 \mathrm{~min}$ & $30 \mathrm{~min}$ & 40min & $20 \mathrm{~min}$ & 30min & 40min \\
\hline Betweenness & \multirow{3}{*}{ Coef } & $\begin{array}{c}1.534 \mathrm{e}-3 \\
(* * *)\end{array}$ & $\begin{array}{c}1.027 \mathrm{e}-3 \\
(* * *)\end{array}$ & $\begin{array}{c}3.862 \mathrm{e}-4 \\
(* * *)\end{array}$ & $\begin{array}{c}2.688 \mathrm{e}-3 \\
(* * *)\end{array}$ & $\begin{array}{c}2.041 \mathrm{e}-3 \\
(* * *)\end{array}$ & $\begin{array}{c}1.157 \mathrm{e}-3 \\
(* * *)\end{array}$ \\
\hline Straightness & & $\begin{array}{c}1.844 \mathrm{e}-4 \\
(* * *)\end{array}$ & $\begin{array}{c}5.144 \mathrm{e}-5 \\
(* *)\end{array}$ & $\begin{array}{c}-3.264 \mathrm{e}-5 \\
(* * *)\end{array}$ & $\begin{array}{c}1.240 \mathrm{e}-4 \\
(* * *)\end{array}$ & $\begin{array}{c}5.740 \mathrm{e}-5 \\
(* *)\end{array}$ & $\begin{array}{c}-3.872 \mathrm{e}-5 \\
(* *)\end{array}$ \\
\hline Closeness & & $\begin{array}{c}1.335 \mathrm{e}+4 \\
\text { (.) }\end{array}$ & $\begin{array}{c}4.950 \mathrm{e}+4 \\
(.)\end{array}$ & $\begin{array}{c}-4.725 e+3 \\
(\text { ) }\end{array}$ & $\begin{array}{c}1.990 \mathrm{e}+4 \\
(* * *)\end{array}$ & $\begin{array}{c}-4.323 e+4 \\
()\end{array}$ & $\begin{array}{c}-9.778 \mathrm{e}+3 \\
()\end{array}$ \\
\hline \multicolumn{2}{|l|}{ R-squared } & 0.08261 & 0.05147 & 0.02821 & 0.1499 & 0.147 & 0.1151 \\
\hline
\end{tabular}

TABLE 2 : Regression of risk severity on network structure measures

For these three measures, most signs of the coefficient of betweenness and straightness corroborate our hypothesis that the higher betweenness and straightness result in a higher risk severity. The exception is at the 40 minute time threshold. The sign of straightness flips when the time threshold increases from 30 to 40 minutes for both accessibility to jobs and to worker. Two reasons may cause such a result. First, straightness tends to be higher for a longer trip since the difference betweens the Euclidian distance and the trip distance is smaller. Second, shortest distance path for a longer trip has a higher possibility that it is not the best route option.

And the signs for the closeness coefficient could not corroborate our hypothesis.

From the $R^{2}$ showed in Table 2, it is obvious that only these three measures do not fully explain risk severity. The model explains 15 percent of the risk severity based on accessibility to workers during the afternoon peak hours at the 20 minutes time threshold, which is the highest $R^{2}$ we achieved. Hence, to explain risk severity, more attributes need to be added in the model, which will be a focus of future research. 
1 CONCLUSION

2 This study applied accessibility as an index to evaluate the risk severity of freeways in the Min-

3 neapolis - St. Paul region based on the TomTom road speed network data for 2010.

The spatial distribution analysis shows that the links with relative higher risk severity are more likely in or near the downtown area and the bridges. The results vary by time threshold, and are more significant at 20 and 30 minute thresholds. The results also vary by time of day.

Betweenness and straightness are significant factors explaining risk severity. And a link with a higher betweenness and straightness tends to have a higher risk severity. The effects of closeness on vulnerability could not be determined based on our current model.

This study assume that the removing of one link would not affect the speed in other links. Future research should test this hypothesis and discover the the speed correlation matrix between links. More attributes of network structure should be considered in future research to better explain risk severity.

\section{REFERENCES}

[1] Chen Anthony, H. K. L., Hai Yang and W. H. Tang, Capacity reliability of a road network: an assessment methodology and numerical results. Transportation Research Part B: Methodological, 2002.

[2] Wakabayashi, H., Reliability Assessment and importance analysis of highway network: a case study of the 1995 Kobe earthquake. In: Proceedings of the First Conference of Hong Kong Society for Transportation Studies, 1996.

[3] Erik Jenelius, T. P. and L.-G. İĹran Mattsson, Importance and exposure in road network vulnerability analysis. Transportation Research Part A, Vol. 40, 2006, pp. 537-560.

[4] D’Este, G. M. and M. A. Taylor, Network vulnerability: an approach to reliability analysis at the level of national strategic transport networks. Network Reliability of Transport. Proceedings of the 1st International Symposium on Transportation Network Reliability (INSTR), 2003.

[5] Lam, W. H. K. and M. L. Tam, Reliability assessment on searching time for parking in urban areas. Network Reliability of Transport, Proceedings of the 1st International Symposium on Transportation Network Reliability (INSTR)., 2003.

[6] Carrion, C. and D. Levinson, Value of travel time reliability: A review of current evidence. Transportation Research Part A, Vol. 46, 2012, pp. 720-741.

[7] Chen Anthony, H. K. L., Hai Yang and W. H. Tang, A capacity related reliability for transportation networks. Journal of advanced transportation, 1999.

[8] Luathep Paramet, H., Agachai Sumalee and F. Kurauchi, Large-scale road network vulnerability analysis: a sensitivity analysis based approach. Transportation, 2011.

[9] Berdica, K., An introduction to road vulnerability: what has been done, is done and should be done. Transport Policy, 2002. 
1 [10] Nicholson, A. and Z. Du, Improving network reliability: a framework. Proceedings of 17th Australian Road Research Board Conference, 1994.

[11] Nicholson A. J., B. M., Schmocker J.-D. and Y. Iida, Assessing transport reliability: malevolence and user knowledge. Proceedings of the 1st International Symposium on Transportation Network Reliability (INSTR), 2003.

[12] AP, T. M., S. V. Sekhar, and G. M. D’Este, Application of accessibility based methods for vulnerability analysis of strategic road networks. Networks and Spatial Economics, 2006.

[13] Taylor, M., An accessibility approach in assessing regional road network vulnerability. Australasian Transport Research Forum (ATRF), , 2008.

[14] TomTom International BV, Instruction of tomtom speed data., 2010, metropolitan Council.

[15] TomTom International BV, Speed Profiles, 2013.

[16] Metropolitan Council, 2010 Transportation Analysis Zone(TAZ) System, 2012.

[17] US Census Bureau, LEHD origin-destination employment statistics dataset structure format version 7.0., 2013.

[18] Taylor, S. V. S., Michael AP and G. M. D'Este, Application of accessibility based methods for vulnerability analysis of strategic road networks. Networks and Spatial Economics, 2006.

[19] Owen, A. and D. Levinson, Access to Destinations: Annual accessibility measure for the Twin Cities Metropolitan Region. Minnesota Department of Transportation Research Services Section, 2012.

[20] Ahmed M. El-Geneidy, D. M. L., Access to Destinations: development of accessibility measures. Minnesota Department of Transportation Research Services Section. Minnesota Department of Transportation Research Services Section. Minnesota Department of Transportation Research Services Section. Minnesota Department of Transportation Research Services Section. Minnesota Department of Transportation Research Services Section, 2006.

[21] Feng, X. and D. Levinson, Evolving Transportation Networks. Springer, 2011.

[22] Xie, F. and D. Levinson, Measuring the structure of road networks. Geographical Analysis, 2006.

[23] Levinson, D., Network structure and city size. PLoS ONE 7, , No. e29721, 2012.

[24] Pavithra Parthasarathi, H. H. and D. Levinson, Network structure and spatial separation. Environment and Planning B: Planning and Design, Vol. 39, 2012, pp. 137-154.

[25] Parthasarathi, P. and D. Levinson, Network Structure and Metropolitan Mobility, 2010, working Paper.

[26] Sevtsuk, A. and M. Mekonnen, Urban network analysis- A new toolbox for ArcGIS, 2012. 
1 [27] Freeman, L. C., A set of measures of centrality based on betweenness. Sociometry, Vol. 40, 2 No. 1, 1977, pp. 35-41.

3 [28] Sergio Porta, P. C. and V. Latora, The network analysis of urban streets: a primal approach. 4 Environment and Planning B: Planning and Design, Vol. 33, 2006, pp. 705-725.

5 [29] Vragovic, E. L. and A. Diaz-Guilera, Efficiency of informational transfer in regular and com6 plex networks. Physical Review E 71, , No. 036122, 2005.

7 [30] Levinson, D. and A. El-Geneidy, The minimum circuity frontier and the journey to work. 8 Regional Science and Urban Economics, Vol. 39, 2009, pp. 732-738.

9 [31] Sabidussi, G., The centrality index of a graph. Psychometrika, Vol. 31, No. 4, 1966. 